\title{
Effect of Mutations in Genes Affecting Homologous Recombination on Restriction Enzyme-Mediated and Illegitimate Recombination in Saccharomyces cerevisiae
}

\author{
ROBERT H. SCHIESTL, ${ }^{1 *}$ JIE ZHU, ${ }^{1}$ AND THOMAS D. PETES ${ }^{2}$ \\ Department of Molecular and Cellular Toxicology, Harvard School of Public Health, Boston, Massachusetts 02115, ${ }^{1}$ \\ and Department of Biology, University of North Carolina, Chapel Hill, North Carolina 27599-3280 2
}

Received 19 November 1993/Returned for modification 27 January 1994/Accepted 29 March 1994

\begin{abstract}
Restriction enzyme-mediated events (REM events; integration of transforming DNA catalyzed by in vivo action of a restriction enzyme) and illegitimate recombination events (IR events; integration of transforming DNA that shares no homology with the host genomic sequences) have been previously characterized in Saccharomyces cerevisiae. This study determines the effect of mutations in genes that are involved in homologous recombination and/or in the repair of double-stranded DNA breaks on these recombination events. Surprisingly, REM events are completely independent of the double-strand-break repair functions encoded by the $R A D 51, R A D 52$, and $R A D 57$ genes but require the $R A D 50$ gene product. IR events are under different genetic control than homologous integration events. In the rad50 mutant, homologous integration occurred at wild-type frequency, whereas the frequency of IR events was 20- to 100 -fold reduced. Conversely, the rad52 mutant was grossly deficient in homologous integration (at least 1,000 -fold reduced) but showed only a 2- to 8-fold reduction in IR frequency.
\end{abstract}

Recombination events involving little or no sequence homology (illegitimate recombination [IR] events) have been observed in many organisms. Such IR events can give rise to genome rearrangements that have been implicated in carcinogenesis (28). Although in the yeast Saccharomyces cerevisiae, integration of DNA sequences into the genome during transformation usually occurs by homologous recombination (16), illegitimate integration events have been recently reported (43, 45). A BamHI URA3 fragment or a linear plasmid containing $U R A 3$ was transformed into a strain lacking any homology to the transforming DNA. Integrated fragments were cloned, and the junction sequences between the transforming DNA and the flanking genomic DNA were determined. The sequences of the target sites were determined by cloning and sequencing of the genomic DNA or by homology search with a DNA sequence data base.

The sequences of these target sites revealed four classes of integration events. When the BamHI enzyme was present in the transformation mixtures, the $U R A 3$ fragments integrated into genomic Bam HI sites. These novel recombination events were termed restriction enzyme-mediated (REM) events (45); similar events were also discovered in Dictyostelium discoideum (22). The simplest interpretation of REM events is that the restriction enzyme enters the cell (perhaps associated with the transforming DNA) and cleaves genomic BamHI sites, allowing the integration of the transforming fragment. In the absence of the Bam HI enzyme, three classes of IR events were found. In one class, insertions resulted from recombination events involving very small amounts of homology (4 bp or less) between the target and the ends of the transforming DNA molecule. In the second class, no such homologies were detected; some fraction of this class may reflect events catalyzed by topoisomerase I (45). For both of these classes, some

\footnotetext{
* Corresponding author. Mailing address: Department of Molecular and Cellular Toxicology, Harvard School of Public Health, 665 Huntington Ave., Boston, MA 02115. Phone: (617) 432-4410. Fax: (617) 432-0377.
}

of the transformants contained target site deletions or duplications from a few base pairs up to more than $1 \mathrm{~kb}$. A third class of transformants resulted from ligation of transforming DNA to yeast mitochondrial DNA fragments located in the nucleus (43). Recombination events between short stretches of homology have also been previously shown to account for recombination events between similar but not identical sequences (29).

Homologous recombination in yeast cells is under the control of many genes. Mutations in genes in the RAD52 epistasis group ( $R A D 50, R A D 51, R A D 52$, and $R A D 57)$ result in defects in double-strand-break repair $(13,37)$ and are defective in meiotic recombination $(33,37)$. Mutations in $R A D 51$ and $R A D 52$ reduce most spontaneous mitotic recombination events, whereas $R A D 50$ is not required for mitotic recombination (27). The RAD52 gene is also essential for UV- and $\gamma$-ray-induced mitotic recombination (36), for homothallic (25, 54) and heterothallic (42) mating-type switching, and for intrachromosomal gene conversion $(19,46)$.

Some recombination events, however, such as unequal sister strand recombination events at the ribosomal DNA locus (35, 56 ), appear to be $R A D 52$ independent. In a rad52 mutant, a double-strand break is efficiently repaired when placed in an array of 18 repeats of $C U P 1$ but not when placed in an array of only three units (31). The effect of rad52 on the integration of transforming DNA is somewhat less clear. Although two groups found little (51) or no (30) effect on integration, two other groups observed large decreases in integration efficiency. Malone et al. (26) found a 50-fold decrease for integration, and Schiestl and Prakash (47) observed a 10,000-fold decrease for integration and a 30 -fold reduction in transformation efficiency of circular plasmids.

The frequency of intrachromosomal recombination is also decreased in a radl mutant $(20,46,53)$. These events are synergistically decreased in the rad1 rad52 double mutant, suggesting that $R A D 1$ and $R A D 52$ work in different pathways for recombination (46), an observation consistent with the classification of these two genes into different epistasis groups. 
This report examines the effects of mutations in $R A D 1$, $R A D 50, R A D 51, R A D 52$, and $R A D 57$ on REM and IR events. We find that REM events are completely independent of the functions encoded by $R A D 1, R A D 51, R A D 52$, and $R A D 57$ but require the $R A D 50$ gene product. IR events are under different genetic control than are homologous integration events.

\section{MATERIALS AND METHODS}

Strains and media. S. cerevisiae RSY12 (MATa leu2-3,112 his3-11,15 ura3::HIS3) was used. This strain lacked URA3 sequences and was constructed by replacement of the entire open reading frame of $U R A 3$ with the HIS3 gene (45). Growth and minimal media were prepared as described previously (39, 49).

Genomic deletions of different $R A D$ genes in the RSY12 genetic background were made by deleting most or all of their open reading frames by the one-step gene disruption method (40). To replace the genomic $R A D 1$ gene from positions -212 to +3853 by the $L E U 2$ gene, we used a $7-\mathrm{kb}$ BamHI DNA fragment containing the $L E U 2$ gene flanked by the $5^{\prime}$ and the 3' RAD1 sequences derived from plasmid pRR46 (38), transforming RSY12 to Leu ${ }^{+}$. The genomic HindIII-SalI RAD50 sequences were replaced by the his $G$ gene of Salmonella typhimurium by a procedure involving several steps. First, an EcoRI fragment derived from pNKY83 (provided by E. Alani and N. Kleckner) (1) containing the $U R A 3$ gene flanked by one copy on each side of the $S$. typhimurium his $G$ gene (which was in turn flanked by the $5^{\prime}$ and $3^{\prime} R A D 50$ sequences) was transformed into RSY12. $\mathrm{Ura}^{+}$transformants were checked for $\mathrm{Rad}^{-}$phenotype, and then $\mathrm{Ura}^{-}$derivatives of the strain were isolated by using 5-fluoro-orotic acid; these derivatives arose by deletion of the $U R A 3$ gene and one copy of the his $G$ gene. The genomic copy of the $R A D 51$ gene was replaced with the LEU2 gene by transformation of RSY12 with a XbaI-PstI fragment from plasmid pDG152 (provided by D. Gietz). Plasmid pDG152 was constructed from plasmid pAM50 (provided by $M$. Aker and R. Mortimer) by deletion of the $2 \mu \mathrm{m}$ yeast origin. The XbaI-PstI fragment contains $R A D 51$ sequences in which the StuI-HpaI fragment was replaced by a HpaI-NruI LEU2 fragment from YEp13. The genomic copy of the $R A D 57$ was replaced with the $L E U 2$ gene as described by Schiestl et al. (48). The genomic RAD52 gene was replaced with the LEU2 gene by transformation of RSY12 with a Bam HI fragment of plasmid pSM20 (kindly provided by D. Schild and $\mathrm{R}$. Mortimer), in which a LEU2 fragment was inserted into the $B g l$ II site within the RAD52 gene.

The phenotype of each mutant was confirmed by testing sensitivity of the cells to $\gamma$ rays ( $R A D 50, R A D 51, R A D 52$, and $R A D 57)$ or UV light (RAD1).

Plasmids. Plasmid pM20 is described in reference 45. Plasmid YEplac195 is a URA3-containing plasmid with a replication origin derived from the $2 \mu \mathrm{m}$ plasmid (11). A derivative of YEplac195 that lacked the unique BamHI site (pJZ195) was prepared by altering the HindIII site in the multicloning site of YEplac195 to a BglII site, treating the resulting plasmid (YEplac195-BglII; constructed by W. Yap) with BamHI and $B g l \mathrm{II}$, and religating the DNA ends. Plasmid pJZ102 was constructed from plasmid pRDG102 (kindly provided by D. Gietz), which contained a 4.8-kb EcoRI-HindIII fragment of the LYS2 gene in the multicloning site of pUC8. The 2.9-kb BamHI-BglII fragment (containing LYS2 sequences) of pRDG102 was replaced with the 1.1-kb BamHI URA3 fragment of plasmid pM20 to yield plasmid pJZ102. Transformation of strain RSY12 with a 3-kb EcoRI-HindIII fragment of pJZ102 (containing the $U R A 3$ gene flanked by $5^{\prime}$ and $3^{\prime}$ sequences of $L Y S 2$ ) resulted in replacement of the genomic $L Y S 2$ gene with the $U R A 3$ gene. The frequency of this transplacement event was used to determine the frequency of homologous integration.

Molecular techniques. Standard methods for molecular biology (41) were used except as noted below. Small-scale plasmid preparations from Escherichia coli were carried out by a modification of the boiling method (17). Cells were collected from a 1-ml overnight culture and resuspended in $0.4 \mathrm{ml}$ of STET buffer $(50 \mu$ l of $1 \mathrm{M}$ Tris- $\mathrm{HCl}$ [pH 7.5], $250 \mu \mathrm{l}$ of $20 \%$ Triton X-100, $160 \mu$ l of $50 \%$ sucrose, $100 \mu$ l of $0.5 \mathrm{M}$ EDTA [pH 8], $440 \mu \mathrm{l}$ of $\left.\mathrm{H}_{2} \mathrm{O}\right) ; 40 \mu \mathrm{l}$ of lysozyme $(10 \mathrm{mg} / \mathrm{ml})$ was added, and the solution was boiled for $60 \mathrm{~s}$, placed on ice, and spun refrigerated for $10 \mathrm{~min}$. The pellet was removed with a toothpick, and $400 \mu \mathrm{l}$ of cold isopropanol was added. After incubation for $10 \mathrm{~min}$ at $-20^{\circ} \mathrm{C}$, the solution was spun for 3 $\mathrm{min}$, and the resulting pellet was resuspended in $50 \mu \mathrm{l}$ of Tris-EDTA and $50 \mu \mathrm{l}$ of $5 \mathrm{M} \mathrm{LiCl}-50 \mathrm{mM}$ Tris- $\mathrm{HCl}$ (pH 8.0). The suspension was incubated on ice for $5 \mathrm{~min}$ and then spun for $5 \mathrm{~min}$ in a microcentrifuge. The supernatant was transferred to a new tube and then precipitated and washed with ethanol; the resulting pellet was dried and dissolved in $60 \mu \mathrm{l}$ of $\mathrm{H}_{2} \mathrm{O}$. A rapid procedure for the preparation of small amounts of large yeast DNA fragments was used (6).

The yeast transformation protocol was described previously $(44,45)$. In this protocol, lithium acetate is used to permeabilize cells and salmon sperm DNA is used as a carrier. Twenty micrograms of CsCl-purified pM20 (46) was treated with $100 \mathrm{U}$ of BamHI (obtained from either New England Biolabs or Life Technologies). After complete digestion, the DNA was precipitated with ethanol and resuspended in $200 \mu l$ of $0.01 \mathrm{M}$ Tris- $\mathrm{HCl}$ (pH 7.5)-0.05 M EDTA-1\% sodium dodecyl sulfate$100 \mu \mathrm{g}$ of proteinase $\mathrm{K}$ per ml. After a 30 -min incubation at $37^{\circ} \mathrm{C}$, the sample was extracted with phenol-chloroformisoamyl alcohol, precipitated with ethanol, washed with $70 \%$ ethanol, and vacuum dried. The pellet was dissolved in water. The yeast cells were then transformed with this solution. For REM events, $100 \mathrm{U}$ of the BamHI enzyme was added together with the plasmid DNA to the transformation mix. All transformation experiments were done at least twice, and the transformation frequencies varied threefold or less in duplicate experiments except for an eightfold difference observed with RSY12/1 $\Delta$.

From 10 yeast transformants with plasmid pM20 inserted at a nonhomologous site, the $U R A 3$ gene and its flanking DNA sequences were recovered by plasmid rescue, and the sequences flanking the URA3 insertions were determined as described previously (45). The junction sequences were compared with sequences in the GenBank DNA sequence data base, and in four cases, the target sites (the genomic sites into which the insertions happened) were found. In the remaining six cases, the junction sequence information was used to design oligonucleotides that could be used to amplify the target sites. DNA was isolated from OD5 (a URA3 derivative of RSY12 [45]), and in separate experiments, pairs of primers designed to produce a DNA fragment of $150 \mathrm{bp}$ in length were used in the PCR (18); amplification of DNA fragments was carried out with a Perkin-Elmer Cetus DNA Thermal Cycler. The sequences $\left(5^{\prime}\right.$ to $\left.3^{\prime}\right)$ of the primers used in these experiments derived from the sequence of individual integrants were as follows: pRS159, CTCGGATCCATCATAGTAATACACAC and GGGAATTCCAACCAGGAGGTAGAT; pRS161, GG GAATTCCATATCTCTTTGCGC and GGAGATCTACAA TTCATGACCACC; pRS171, ATCCTGTGGATCCGTTAAC and GGCAAGAGAATTCAATTGGAC; pRS173, GTTGA ATTCAAAGAACGGCGT and ACCGGATCCCAATGAA 
TABLE 1. Frequency of REM events in $R A D^{+}, \operatorname{rad} 1 \Delta, \operatorname{rad} 50 \Delta$,

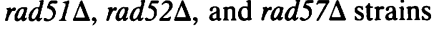

\begin{tabular}{|c|c|c|c|c|c|}
\hline \multirow[t]{2}{*}{ Strain } & \multirow[t]{2}{*}{ Genotype } & \multirow{2}{*}{$\begin{array}{c}\text { No. of } \\
\text { REM } \\
\text { events/ } \mu g \\
\text { of DNA }{ }^{a}\end{array}$} & \multicolumn{2}{|c|}{$\begin{array}{c}\text { Proportion of } \\
\text { transformants } \\
\text { with insertions in } \\
\text { Bam HI sites }\end{array}$} & \multirow{2}{*}{$\begin{array}{l}\text { Bam HI-increased } \\
\text { transformation } \\
\text { efficiency }\end{array}$} \\
\hline & & & $\begin{array}{l}\text { BamHI } \\
\text { absent }^{d}\end{array}$ & $\begin{array}{l}\text { BamHI } \\
\text { present }^{e}\end{array}$ & \\
\hline RSY12 & $R A D^{+}$ & $10.3(5.1)$ & $2 / 39$ & $34 / 46^{f}$ & 7 \\
\hline RSY12/1s & $\mathrm{radl} \Delta$ & $12.2(3.0)$ & $1 / 7$ & $10 / 10$ & 12 \\
\hline RSY12/50 & $\mathrm{rad} 50 \Delta$ & $0.1(0.7)$ & $1 / 3$ & $1 / 4$ & 1 \\
\hline RSY12/51D & $\operatorname{rad51\Delta }$ & $9.5(2.5)$ & $0 / 12$ & $10 / 14$ & 15 \\
\hline RSY12/52 & $\operatorname{rad52\Delta }$ & $2.6(8.0)$ & $1 / 10$ & $21 / 21$ & 32 \\
\hline RSY12/57D & $\operatorname{rad57\Delta }$ & $12.6(18.4)$ & $0 / 14$ & $12 / 12$ & 24 \\
\hline
\end{tabular}

${ }^{a}$ Transforming DNA was pM20. Each value in parentheses is the number of REM events per $10^{4}$ transformants with plasmid YEplac195 ( $2 \mu \mathrm{m}$, URA3 [11]) of the same culture; this procedure normalizes for differences in transformation efficiency in different cultures.

${ }^{b}$ DNA was isolated from yeast transformants, treated with BamHI, and examined by Southern analysis. The number of transformants that had a URA3 $B a m \mathrm{HI}$ fragment of $1.2 \mathrm{~kb}$ (REM events) as a fraction of total transformants is shown.

${ }^{c}$ Expressed as the transformation frequency with Bam $\mathrm{HI}$ divided by the transformation frequency without $B a m \mathrm{HI}$.

${ }^{d}$ The BamHI enzyme was removed prior to transformation.

e The BamHI enzyme was removed and then readded to the purified BamHI fragments prior to transformation.

$f$ Data from reference 45 .

CTT; pRS198, CTTATCTTCGCTAGACATTC and AC GAGGATCCTGTCGTTTCTT; and pRS199, TGTGGATC CGTTCCGAACTT and GGTAGCCAGAACTTCTCATC. Following amplification, the resulting fragments were sequenced directly (protocol from U.S. Biochemical Corporation).

\section{RESULTS}

Previously, we found that a restriction fragment containing $U R A 3$ or a linear plasmid containing $U R A 3$ is able to integrate into the yeast genome in the absence of any gross sequence homology between the transforming DNA and the yeast genome $(43,45)$. When the transforming solution contained the $B a m H I$ restriction enzyme, the $U R A 3$ fragment integrated into genomic Bam HI sites (REM events). In the absence of the restriction enzyme, the fragment integrated into the genome by a variety of types of IR.

To further characterize REM and IR events, we investigated the effects of mutations in genes involved in homologous recombination. All strains were isogenic to RSY12, the strain that lacked URA3 sequences which was used in the previous studies. The mutant strains were constructed by deletion of most of the open reading frames of the genes RAD1, RAD50, $R A D 51, R A D 52$, and $R A D 57$. The integrating DNA fragment was a 1.1-kb Bam HI fragment derived from pM20 that included only yeast sequences.

REM events in wild-type, rad1, rad51, rad52, and rad57 strains. The effects of various rad mutations on REM events are shown in Table 1. In these experiments, plasmid pM20 was digested with Bam HI and transformed into RSY12 with the BamHI restriction enzyme. To control for transformation efficiency, we transformed the same batches of competent cells with YEplac195, a URA3-containing plasmid with a $2 \mu \mathrm{m}$ plasmid origin. Table 1 shows the number of REM events per microgram of transforming DNA and the number of REM events per $10^{4}$ transformants of YEplac195. Transformants were diagnosed as resulting from REM events by Southern analysis of a genomic BamHI digest; insertion of the transforming 1.1-kb Bam HI $U R A 3$ fragment should result in a single $1.1-\mathrm{kb}$ Bam HI fragment in a genomic digest if the fragment was inserted into a genomic BamHI site. No effect on the efficiency of REM events was found for rad1, rad51, rad52, and rad57 mutants compared with the wild type. The apparent lower number of REM events per microgram of pM20 DNA in the rad52 mutant is due to the lower overall transformation frequency in that strain. Relative to the frequency of transformation in the absence of the BamHI enzyme (IR events), the addition of the BamHI enzyme caused an increase in transformation frequency in all of these strains. The rad52 mutant showed the most dramatic increase (32-fold compared with the 7 -fold increase in the wild type). As discussed below, this increase reflects a decrease in the frequency of IR events in rad52 strains.

In contrast to the results obtained with the other mutants, the rad50 mutation reduced (or eliminated) REM events. The frequency of transformation was not elevated by adding the restriction enzyme to the transformation reaction in the rad50 strain, and most (three of four) of the transformants did not have the transforming fragment integrated into a genomic BamHI site. Interpretations of this result will be given in Discussion.

High-level expression of the EcoRI endonuclease in yeast cells causes lethality that is more pronounced in a rad52 mutant strain (3). Since we found successful REM events in the rad52 mutant, we determined cell survival after exposure to the restriction enzyme. For both wild-type and rad52 strains, 20 to $50 \%$ of the cells survived the transformation procedure. If we normalize the survival frequency of wild-type strains to 1.0 , then the survival of the rad52 strain was 0.9 in the absence of the enzyme and 0.84 in the presence of the enzyme. Although this result indicates that there is no gross effect of the restriction enzyme on the survival of a rad52 strain, there could be a difference in the survival of those cells in the population that are transformation competent. To test this possibility, we transformed the rad52 strain with plasmid pJZ195 (a derivative of the autonomously replicating plasmid YEplac195 that lacked a BamHI restriction site), in the presence and the absence of the BamHI enzyme. The frequency of transformation was approximately the same, $9.0 \times 10^{3}$ transformants per $\mu \mathrm{g}$ in the absence of the enzyme and $9.3 \times 10^{3}$ transformants per $\mu \mathrm{g}$ in its presence (average of two experiments). Thus, transformable rad52 cells are not killed by the restriction enzyme. Although we cannot exclude the possibility that most of the cells that take up DNA do not take up the restriction enzyme, we favor the interpretation that rad52 strains are capable of repairing double-strand breaks caused by Bam HI. Alternative interpretations will be outlined in Discussion.

IR events in $R A D^{+}, \operatorname{radl}, \operatorname{rad50}, \mathrm{rad51}, \mathrm{rad52}$, and rad57 strains. To score IR events, we also used BamHI-treated pM20 DNA. After using proteinase $K$ and phenol to remove the restriction enzyme, the DNA was transformed into wild-type and rad mutant strains. The frequencies of IR events are shown in Table 2. With the same batches of cells used in the IR transformation experiments, two other types of transforming DNA samples were used as controls. First, as in the REM experiments, we transformed the cells with YEplac195, a plasmid capable of autonomous DNA replication. This control monitors the permeability of the cell (and its nucleus) to transforming DNA and the recovery of the cell from the trauma of the transformation procedure; we assume that those mutants that have reduced permeability and/or recovery for YEplac195 have comparably reduced permeability and/or recovery for pM20 DNA. For the second control, we trans- 
TABLE 2. Frequency of IR relative to homologous integration events in $R A D^{+}, \operatorname{rad} 1 \Delta, \operatorname{rad} 50 \Delta, \operatorname{rad} 51 \Delta, \operatorname{rad} 52 \Delta$, and $\operatorname{rad} 57 \Delta$ strains

\begin{tabular}{|c|c|c|c|c|c|}
\hline \multirow[b]{2}{*}{ Strain } & \multirow[b]{2}{*}{ Genotype } & \multicolumn{3}{|c|}{ No. of transformants/ $\mu \mathrm{g}$ of $\mathrm{DNA}^{a}$} & \multirow[b]{2}{*}{ IR/HI ratio ${ }^{b}$} \\
\hline & & YEplac195c & $\begin{array}{l}\text { IR, BamHI- } \\
\text { treated pM20 }\end{array}$ & $\mathrm{HI}, \mathrm{pJZ102} 2^{e}$ & \\
\hline RSY12 & $R A D^{+}$ & 17,000 & $18.2(11)$ & $408(240)$ & $0.05(1)$ \\
\hline RSY12/1 $\Delta$ & $\operatorname{rad} 1 \Delta$ & 27,000 & $6.5(2.4)$ & $164(61)$ & $0.04(0.9)$ \\
\hline RSY12/50 & $\operatorname{rad} 50 \Delta$ & 5,800 & $0.07(0.1)$ & $264(455)$ & $0.0003(0.006)$ \\
\hline 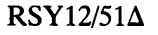 & $\operatorname{rad51\Delta }$ & 19,000 & $1.0(0.5)$ & $60(32)$ & $0.02(0.4)$ \\
\hline RSY12/52 $\Delta$ & $\operatorname{rad} 52 \Delta$ & 1,400 & $0.2(1.4)$ & $<0.03(<0.2)^{f}$ & $>7(>156)$ \\
\hline RSY12/57D & $\operatorname{rad57\Delta }$ & 11,900 & $1.2(1.0)$ & $26(22)$ & $0.04(1.0)$ \\
\hline
\end{tabular}

${ }^{a}$ Average of two experiments with 10 to $50 \mu \mathrm{g}$ of DNA for each experiment and each plasmid. Each value in parentheses is the number of integration events per $10^{4}$ YEplac195 transformants to normalize for transformation efficiency.

${ }^{b}$ Number of IR events (pM20, URA3 fragment) divided by the number of homologous integration (HI) events in the same strain. Each value in parentheses is the IR/HI ratio for the mutant strain divided by the IR/HI ratio for the wild type.

c Plasmid YEplac195 (11) was used to assay the transformability of the culture.

${ }^{d}$ Plasmid pM20 was digested at two sites with BamHI to release a 1.2-kb URA3 fragment for transformation.

e pJZ102 was digested with EcoRI and HindIII to release a URA3 fragment that was flanked by the 5' and 3' ends of LYS2; this fragment integrates into the LYS2 locus.

${ }^{f}$ Only two Ura ${ }^{+}$transformants were obtained in these experiments. Since both of these transformants were Lys ${ }^{+}$, they do not represent homologous integration. In contrast, about 98 to $99 \%$ of all $\mathrm{Ura}^{+}$colonies from the other strains were $\mathrm{Lys}^{-}$.

formed the cells with an EcoRI-HindIII fragment (URA3 flanked by $L Y S 2$ sequences) derived from pJZ102. When a $R A D^{+}$strain was transformed with this fragment, $98 \%$ of the $\mathrm{Ura}^{+}$transformants were $\mathrm{Lys}^{-}$, as expected if this fragment integrates by homologous recombination at the LYS2 locus.

As shown in Table 2 , in the wild-type strain, homologous integration was about 40 -fold less frequent than transformation with an autonomously replicating plasmid, and IR events were about 20 -fold less frequent than homologous integration events. In the radl mutant, the frequencies of IR, as well as homologous integration, were reduced about 2.5 -fold, resulting in a ratio of IR to homologous integration events that was similar to that observed for wild-type (0.045). Similarly, mutations in $R A D 51$ and $R A D 57$ caused a decrease of about 10 -fold in both IR and homologous integration events, resulting in ratios similar to the wild-type ratio. In two strains, however, rad mutations had dramatically different effects on the two types of integration. In the rad50 strain, homologous integration was unaffected by the mutation, whereas the frequency of IR events was reduced substantially (about 100-fold). The rad52 mutation had the opposite effect, reducing homologous integration (1,000-fold reduction) much more completely than IR events (8-fold reduction).

Target sites for IR events in the rad52 and RAD52 strains. As described in the introduction, three types of IR events have been observed: those involving microhomologies between the target and ends of the transforming DNA (the major class), those that involve integration without homology, and those that involve ligation of the transforming DNA to mitochondrial DNA fragments $(43,45)$. One interpretation of the modest reduction in IR events in the rad52 mutant is that IR events involving microhomologies were eliminated by the mutation. To test this possibility, we characterized the target sites of IR events from the rad52 deletion strain and compared them with the target sites from the wild-type strain. To identify the sequences flanking the insertion, we treated DNA from the transformed cells with a restriction enzyme (usually BglII) that does not cut within the $U R A 3$ fragment. These genomic fragments were ligated to a vector (YEplac181) cleaved to generate a fragment with compatible ends (other technical details are described in reference 45). The ligated DNA fragments were transformed into the yeast strain RSY12 to yield $\mathrm{Ura}^{+}$transformants, and plasmids were rescued from these transformants into $E$. coli. The DNA sequences of the genomic junctions were determined with primers homologous to the ends of the URA3 fragment. To determine the target sites of the IR events, we compared the junction sequences with existing GenBank sequences. In the cases in which no match has been found, the information from the junction sequences was used to design primers for amplification of the target sites in the RSY12 strain that did not contain an insertion. The same primers were used to sequence the target sites from the PCR-amplified products.

The target sites from the wild-type strain are shown in Fig. 1a, and the target sites from the rad52 strain are shown in Fig. 1b. For five of the six integrants shown in Fig. 1a, the genomic site contained only the $1.1-\mathrm{kb} U R A 3$ fragment derived from pM20. In one integrant (represented by plasmid pRS166), the genomic site contained both URA3 and pUC-derived sequences. We interpret this integrant to reflect partial cleavage of the transforming pM20 DNA. As reported previously (43, 45 ), the target sites in wild-type strains indicate that often (five of six integrants), there are microhomologies between the ends of the transforming DNA molecule and the target. In some integrants, these end-directed events involve both ends (for example, those represented by pRS159 and pRS161) whereas in other integrants (pRS162), only one end shows this type of homology. In one transformant (pRS180), no homology was detected between target and transforming DNA. One of the ends of this transformant is contiguous with CTT. As discussed previously, this class of integrant may reflect topoisomerase I-catalyzed insertion of transforming DNA (44), since CTT is one of the preferred sites for cleavage by this enzyme $(4,34)$.

In most of the integrants, the two ends of the transforming DNA fragment appear to attack the same bases in the target (pRS159 and pRS161) or nearby bases (pRS181 and pRS180). In two transformants (pRS162 and pRS166), however, the two ends apparently independently invaded targets within the chromosome. For the transformant represented by plasmid pRS162, one end of the plasmid is contiguous with the MRF1 gene (33) and the second end is contiguous with the PAP1 gene (23). Thus, insertion of the transforming DNA is likely to be associated with a DNA rearrangement such as a deletion, translocation, or duplication; since the relative genomic locations of $M R F 1$ and $P A P 1$ are not known, we do not know which of these alterations is most likely. In previous studies (43), we found an IR event that was associated with a duplication of the YEF3 gene. 


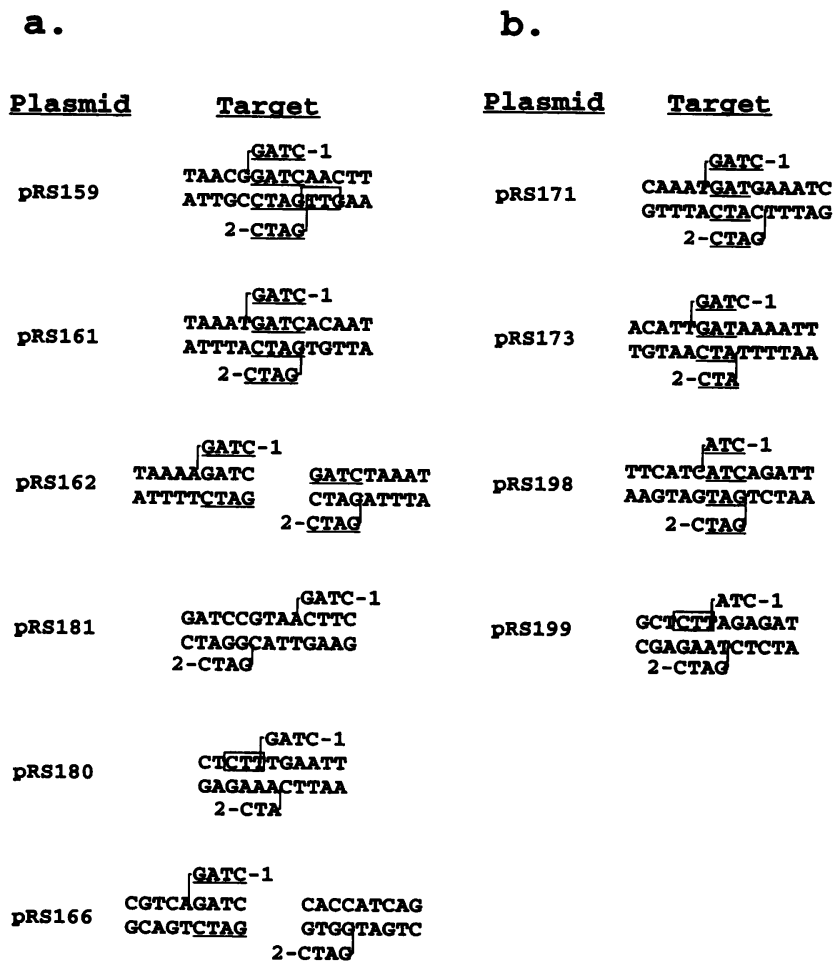

FIG. 1. Flanking DNA sequences and targets of IR events of a BamHI URA3 fragment in wild-type (RSY12) and rad52 (RSY12/52A) strains. As described in Materials and Methods, plasmid pM20 was treated with BamHI, the restriction enzyme was removed, and the resulting DNA fragments were used for transformation. The URA3 gene was rescued from individual transformants, and about $200 \mathrm{bp}$ of DNA flanking the insertion were sequenced. For 9 of the 10 transformants, only the 1.2-kb BamHI fragment derived from pM20 was inserted; in one integrant (pRS166), all of the pM20 sequences were inserted as a single Bam HI fragment, presumably as a consequence of partial digestion of the plasmid. Target sites (yeast genomic sequences without the insertion) were either PCR generated or obtained by identification of the target in GenBank. From the sequence of the target sites and junctions, we inferred the position of the insertion. The DNA sequence of the target is shown as double-stranded DNA. The sequences shown above and below the target represent the sequences of the ends of the transforming DNA. For most of the insertions, the first bases derived from the transforming DNA were GATC, as expected if the transforming DNA integrated without any degradation, since Bam HI generates ends with a protruding GATC sequence. For some of the insertions (for example, that represented by pRS180), a single base was lost from one end of the transforming DNA. The vertical lines drawn between bases in the target sequences and the ends of the transforming DNA represent the predicted sites at which the targets were cleaved to allow integration of the transforming DNA. The numbers adjacent to GATC in the transforming DNA reflect the sequences near the $5^{\prime}(1)$ and 3' (2) ends of URA3. The underlined regions in the target and fragment ends represent homology between the plasmid ends and the target site; we show only those homologies involving more than two base pairs immediately adjacent to the point of insertion of the transforming fragment. The boxed regions in the target represent CTT or GTT (consensus topoisomerase I binding sites) adjacent to the cleavage sites. (a) Integration events in the $R A D^{+}$ strain RSY12. Targets represented by plasmids pRS159 and pRS161 were generated by sequence analysis of PCR products generated from the untransformed strain (as described in Materials and Methods). In the transformant represented by pRS162, one end of the transforming fragment was inserted into the $P A P 1$ gene (GenBank accession number X60307), whereas the other end was inserted into the MRF1 gene (accession number X60381). The insertions represented by pRS181 and pRS166 were in ribosomal DNA (accession numbers J01355 and X03728, respectively), and that represented by pRS180 was
Target sites were obtained for four IR events in the rad52 strain (Fig. 1b). In three of these four integrants, microhomologies were found between both ends of the transforming DNA and the targets. In these integrants, only three of the four bases matched instead of the four-of-four matches detected in the wild-type strain. It is unlikely that this difference represents a fundamental distinction between IR events in rad52 and in wild-type cells, since IR events involving three of four bases have also been observed in wild-type cells (43), using a different type of transforming DNA (URA3 sequences embedded in pUC sequences). In summary, with the $U R A 3$ gene derived from pM20, the target specificities for IR events appear similar in rad52 and wild-type strains.

\section{DISCUSSION}

In this study, we examined the effects of mutations in various genes affecting homologous recombination on REM and IR events. Below, we will primarily emphasize three points. First, repair of the DNA lesion responsible for REM events (presumably, a double-strand break) does not require the $R A D 51$, $R A D 52$, or $R A D 57$ gene product, all of which are proteins that are required for the repair of double-strand breaks caused by other agents. Second, REM events require the $R A D 50$ gene product. Third, the genetic control of IR events is different from the control of integration of transforming DNA sequences that share homology to the genome.

Effects of rad mutations on REM events. The lack of effect of the rad51, rad52, and rad57 mutations on REM events is a surprising result, since these genes have been shown to be involved in the repair of many different types of double-strand breaks in yeast cells, and REM events are likely to involve a double-strand break catalyzed by the restriction enzyme. Strains with rad51, rad52, or rad57 mutations are extremely sensitive to $\gamma$ rays (which cause double-strand breaks; for reviews, see references 13 and 37) and in homologous recombination induced by $\gamma$ rays (36). Double-strand breaks caused by cleavage of the $M A T$ locus by the $\mathrm{HO}$ endonuclease are unrepaired in a rad52 genetic background, leading to cell death (24); rad51 is also required for mating-type switching $(33,37)$. Barnes and Rine (3) showed that production of EcoRI in yeast cells by using a strong promoter caused lethality in a rad52 genetic background. In summary, in previous studies, the repair of three different types of double-strand breaks ( $\gamma$ ray induced, HO endonuclease induced, and EcoRI induced) appear defective in strains mutant for rad52; rad51 strains are also defective in the repair of the first two types of breaks.

Two factors relevant to the lack of the effects of rad52 and rad51 on REM events are (i) the type of double-strand break and (ii) the amount of double-strand breaks. The exact nature of the double-strand breaks induced by $\gamma$ rays is not known; however, it is clear that $\gamma$ rays cause DNA lesions, in addition to double-strand breaks, that may be relevant to $\gamma$-ray-induced cell death. For example, $\gamma$ rays also cause DNA nicks and base adducts and produce free radicals that can oxidize proteins (10). In addition, DNA double-strand breaks caused by $\gamma$ rays can release a base and leave blunt termini with $5^{\prime}$-phosphoryl and $3^{\prime}$-phosphoryl or $3^{\prime}$ phosphoglycolate ends that cannot be ligated (15). Cleavage of DNA by $\mathrm{HO}$ endonuclease in vitro

in the VPS35 gene (accession number S42186). (b) Integration events in the rad52 strain RSY12/52 . Target sites for all four genomic insertions were obtained by sequence analysis of PCR-generated products as described in Materials and Methods. 
results in 4-bp protruding $3^{\prime}$ ends (21), although it is not clear whether these ends are free or protected by cellular proteins in vivo. For both EcoRI and BamHI, the DNA lesion is a double-strand break with a 4-bp protruding $5^{\prime}$ single strand. The second relevant factor may be the level of double-strand breaks. In our experiments, we have no direct measurement of the number of cells that take up the restriction enzyme or of the amount of enzyme taken up. It is likely, however, that the amount of restriction enzyme per cell in our experiments is considerably less than that in the experiments of Barnes and Rine (3).

Considering the two factors described above, there are two plausible interpretations of our data. One possibility is that yeast cells can repair double-strand breaks induced by restriction enzymes with reasonable efficiency, perhaps by simple ligation. When a very large number of breaks are introduced, as in the experiments of Barnes and Rine (3), this system cannot repair all of the breaks and cell lethality is observed. Alternatively, it is possible that the BamHI-catalyzed insertion of transforming DNA proceeds through an intermediate that does not involve free ends. For example, the ends produced by cleavage with Bam HI may be held together by the structure of the chromatin, although perhaps tagged in some way allowing the introduction of the transforming DNA with compatible ends. A related possibility is that the Bam $\mathrm{HI}$ enzyme acts in the yeast cells as a site-specific recombination enzyme, integrating the transforming DNA without producing free ends; recently, a number of similarities have been detected between certain restriction enzymes and enzymes that catalyze site-specific recombination (5).

Experiments have been done in $E$. coli with results strikingly similar to those that we have observed in $S$. cerevisiae. Heitman et al. (14) found that lexA or $\operatorname{rec} A$ mutants are no more sensitive than wild-type cells to in vivo scission of DNA by the EcoRI endonuclease, although ligase mutants were extremely sensitive to this type of break. These workers suggested that the two ends resulting from EcoRI cleavage may be held together by the structural elements of the chromosome or other DNA-binding proteins.

In contrast to the effects of the other rad mutants tested, the rad50 mutation significantly decreased the level of REM events. It is possible that the $R A D 50$ gene product promotes REM events by stabilizing interactions involving very small amounts of homology. We will discuss this interpretation in more detail when the role of $R A D 50$ in IR events is examined.

Effects of rad mutations on IR events. The rad51, rad52, and rad57 mutations reduced the frequency of IR events about 20-, $10-$, and 10-fold, respectively. The sequence of the integration sites in rad52 strains indicated that the mutation did not eliminate integration events involving microhomologies between the ends of the transforming DNA and the target site. The rad51 and rad57 mutations had relatively small effects on homologous integration (8- and 10 -fold reductions, respectively), whereas rad52 virtually eliminated this type of recombination (1,000-fold reduction). In summary, neither rad51, rad52, nor rad57 eliminates IR events, although all three mutations result in lower frequencies of this class of event. The rad52 mutation affects recombination events that involve extensive sequence homology more dramatically than those events (IR events) that involve microhomologies.

It has been previously established that mutations in $R A D 51$, $R A D 52$, or $R A D 57$ lead to deficiencies in most $(33,37)$ but not all $(31,35,56)$ types of spontaneous mitotic and meiotic recombination. Although the precise role of the gene products encoded by these genes has not been determined, the Rad51p shows structural and functional similarities to the RecA pro- tein (50); furthermore, the $\operatorname{Rad} 51 \mathrm{p}$ protein binds to the Rad52p protein. Thus, it has been proposed that these two proteins may be involved in either the homology search step or strand exchange step of recombination (50). White and Haber (55) found that broken DNA ends formed by $\mathrm{HO}$ endonuclease in vivo were more extensively degraded in rad52 strains than in wild-type strains and suggested that $R A D 52$ function might be required for catalyzing strand invasion. Our results are consistent with the conclusion that IR events also are stimulated by the $R A D 51-R A D 52$ complex, presumably because this complex initiates or stabilizes strand invasion.

IR events were originally defined as recombination events that occur in the absence of $\operatorname{Rec} A$ in $E$. coli (9). Short repeated sequences in $E$. coli often are hot spots for deletions that occur at a frequency that is $\operatorname{Rec} A$ independent (reviewed by Ehrlich [7]). The microhomology-mediated IR events appear analogous to IR events in bacteria. Since these events in yeast cells are at least partly dependent of $R A D 52$ and $R A D 51$, it is possible that the mechanism involved in this type of recombination is somewhat different from that observed in bacteria.

In our experiments, the rad1 mutation had little effect on REM and IR events (Tables 1 and 2). The RADI gene is involved in excision repair and in some forms of homologous recombination. Specifically, deletion events between duplicated sequences in mitosis are $R A D 1$ dependent $(20,46,52)$. Interchromosomal recombination and meiotic recombination events, however, are not dependent on RAD1 (46). FishmanLobell and Haber (8) showed that the $R A D 1$ gene product is likely to be an endonuclease that has a role in recombination events in which nonhomologous DNA sequences are removed from the ends of the interacting DNA molecules. Our experiments indicate that this activity is not required for REM or IR events. In IR events in wild-type strains, most integration events involve no loss or very little loss of bases from the transforming DNA, indicating that the $R A D 1$ gene product is relatively inactive on this type of substrate.

Strains with the rad50 mutation had approximately wild-type levels of homologous integration (2-fold elevated relative to the wild-type level) but severely reduced (about 85 -fold) levels of IR events. Most mutants of rad50 are deficient in repair of damage induced by $X$ rays and in meiotic recombination (reviewed by Petes et al. [33]). Null mutants of $R A D 50$ fail to produce the meiosis-specific double-strand breaks that are thought to represent the initiating lesion for meiotic exchange (2). Strains with the mutant allele rad50S can produce meiosisspecific double-strand breaks but are incapable of processing these breaks into recombinogenic lesions (2). Strains with rad50 mutations also slowly process double-strand breaks induced in mitotic cells (52). In contrast to the strong meiotic $\mathrm{Rec}^{-}$phenotype, rad50 mutants generally have slightly elevated levels of spontaneous mitotic recombination $(12,27)$. Mating-type switching also occurs independently of $R A D 50$ $(13,33,37)$. Given the complicated (and sometimes contrary) effects of rad50 on recombination and repair, it is difficult to give a single explanation for its cellular role; since some mutations of the protein affect meiotic recombination without affecting DNA repair (2), it is likely that the protein has domains with different roles. One explanation for the differential effects on homologous integration and IR events in our experiments is that the $R A D 50$ gene product specifically stabilizes interactions involving very small regions of homology. This possibility is supported by the observation that the $R A D 50$ gene product is required for REM events. Given the complicated phenotypes associated with rad50 mutations, it is also possible that the primary role of the $R A D 50$ gene product is to target other proteins (such as strand transferases, ligase, and 
nucleases) to DNA lesions. Whatever the reason for its effect, $R A D 50$ is the first identified gene product that appears to affect illegitimate recombination without affecting normal mitotic exchange. If mammalian homologs of this gene are identified, it may be possible to design cell lines allowing more efficient targeting of transforming DNA to the homologous chromosomal location by using antisense or other methods to inhibit $R A D 50$ gene function.

\section{ACKNOWLEDGMENTS}

We thank Margaret Dominska and Nick Carls for excellent technical assistance. We also thank $\mathrm{H}$. Klein, members of the Schiestl and Petes laboratories, and the reviewers for comments on the manuscript.

This research was supported by grant $\mathrm{CN}-83$ from the American Cancer Society to R.H.S. and by NIH grant GM47256 to T.D.P.

\section{REFERENCES}

1. Alani, E., L. Cao, and N. Kleckner. 1989. A method for gene disruption that allows repeated use of URA3 selection in the construction of multiply disrupted yeast strains. Genetics 116:541545.

2. Alani, E., R. Padmore, and N. Kleckner. 1990. Analysis of wild type and rad50 mutants of yeast suggests an intimate relationship between meiotic chromosome synapsis and recombination. Cell 61:419-436.

3. Barnes, G., and J. Rine. 1985. Regulated expression of endonuclease EcoRI in Saccharomyces cerevisiae: nuclear entry and biological consequences. Proc. Natl. Acad. Sci. USA 82:1354 1358.

4. Been, M. D., R. R. Burgess, and J. J. Champoux. 1984. Nucleotide sequence preference at rat liver and wheat germ type 1 DNA topoisomerase breakage sites in duplex SV40 DNA. Nucleic Acids Res. 12:3097-3114.

5. Conrad, M., and M. D. Topal. 1989. DNA and spermidine provide a switch mechanism to regulate the activity of restriction enzyme NaeI. Proc. Natl. Acad. Sci. USA 86:9707-9711.

6. Denis, C. L., and E. T. Young. 1983. Isolation and characterization of the positive regulatory gene ADRI from Saccharomyces cerevisiae. Mol. Cell. Biol. 3:360-370.

7. Ehrlich, S. D. 1989. Illegitimate recombination in bacteria, p. 799-832. In D. E. Berg and M. M. Howe (ed.), Mobile DNA. American Society for Microbiology, Washington, D.C.

8. Fishman-Lobell, J., and J. E. Haber. 1992. Removal of nonhomologous DNA ends in double-strand break recombination: the role of the yeast ultraviolet repair gene $R A D I$. Science 258:480-484.

9. Franklin, N. 1971. Illegitimate recombination, p. 175-194. In A. D. Hershey (ed.), The bacteriophage lambda. Cold Spring Harbor Laboratory, Cold Spring Harbor, N.Y.

10. Friedberg, E. C. 1985. DNA repair. W. H. Freeman, New York.

11. Gietz, R. D., and A. Sugino. 1988. New yeast-Escherichia coli shuttle vectors constructed with in-vitro-mutagenized yeast genes lacking six-base-pair restriction sites. Gene 74:527-534.

12. Gottlieb, S., J. Wagstaff, and R. E. Esposito. 1989. Evidence for two pathways of meiotic intrachromosomal recombination in yeast. Proc. Natl. Acad. Sci. USA 86:7072-7076.

13. Haynes, R. H., and B. Kunz. 1981. DNA repair and mutagenesis in yeast, p. 371-414. In J. Strathern, E. Jones, and J. R. Broach (ed.), The molecular biology of the yeast Saccharomyces cerevisiae: life cycle and inheritance. Cold Spring Harbor Laboratory, Cold Spring Harbor, N.Y.

14. Heitman, J., N. D. Zinder, and P. Model. 1989. Repair of the Escherichia coli chromosome after in vivo scission by EcoRI endonuclease. Proc. Natl. Acad. Sci. USA 86:2281-2285.

15. Henner, W. D., L. Rodriguez, S. M. Hecht, and W. A. Haseltine. 1983. Gamma ray induced deoxyribonucleic acid strand breaks, $3^{\prime}$ glycolate termini. J. Biol. Chem. 258:711-713.

16. Hinnen, A., J. B. Hicks, and G. R. Fink. 1978. Transformation of yeast. Proc. Natl. Acad. Sci. USA 75:1929-1933.

17. Holmes, D. S., and M. Quigley. 1981. A rapid boiling method for the preparation of bacterial plasmids. Anal. Biochem. 114:193197.
18. Innis, M. A., D. H. Gelfand, J. J. Sninsky, and T. J. White. 1990. PCR protocols: a guide to methods and applications. Academic Press, New York.

19. Jackson, J. A., and G. R. Fink. 1981. Gene conversion between duplicated genetic elements in yeast. Nature (London) 292:306311.

20. Klein, H. L. 1988. Different types of recombination events are controlled by the RADI and RAD52 genes of Saccharomyces cerevisiae. Genetics 120:367-377.

21. Kostriken, R., J. N. Strathern, A. J. S. Klar, J. B. Hicks, and F. Heffron. 1983. A site-specific endonuclease essential for matingtype switching in Saccharomyces cerevisiae. Cell 35:167-174.

22. Kuspa, A., and W. F. Loomis. 1992. Tagging developmental genes in Dictyostelium by restriction enzyme mediated integration of plasmid DNA. Proc. Natl. Acad. Sci. USA 89:8803-8807.

23. Lingner, J., J. Kellermann, and W. Keller. 1991. Cloning and expression of the essential gene for poly(A) polymerase from $S$. cerevisiae. Nature (London) 354:496-498.

24. Malone, R. E., and R. E. Esposito. 1980. The RAD52 gene is required for homothallic interconversion of mating types and spontaneous mitotic recombination in yeast. Proc. Natl. Acad. Sci. USA 77:503-507.

25. Malone, R. E., and R. E. Esposito. 1981. Recombinationless meiosis in Saccharomyces cerevisiae. Mol. Cell. Biol. 1:891-901.

26. Malone, R. E., B. Montelone, C. Edwards, K. Carney, and M. F. Hoekstra. 1988. A reexamination of the role of the $R A D 52$ gene in spontaneous mitotic recombination. Curr. Genet. 14:211.

27. Malone, R. E., T. Ward, S. Lin, and J. Waring. 1990. The RAD50 gene, a member of the double-strand break epistasis group, is not required for spontaneous mitotic recombination in yeast. Curr. Genet. 18:111-116.

28. Meuth, M. 1989. Illegitimate recombination in mammalian cells, $p$. 833-860. In D. E. Berg and M. M. Howe (ed.), Mobile DNA. American Society for Microbiology, Washington, D.C.

29. Mezard, C., D. Pompon, and A. Nicolas. 1992. Recombination between similar but not identical DNA sequences during yeast transformation occurs within short stretches of identity. Cell 70:659-670.

30. Orr-Weaver, T. L., J. W. Szostak, and R. J. Rothstein. 1981. Yeast transformation: a model system for the study of recombination. Proc. Natl. Acad. Sci. USA 78:6354-6358.

31. Ozenberger, B., and G. S. Roeder. 1991. A unique pathway of double-strand break repair operates in tandemly repeated genes. Mol. Cell. Biol. 11:1222-1231.

32. Pel, H. J., C. Maat, M. Rep, and L. A. Grivell. 1992. The yeast nuclear gene $M R F 1$ encodes a mitochondrial peptide chain release factor and cures several mitochondrial RNA splicing defects. Nucleic Acids Res. 20:6339-6346.

33. Petes, T. D., R. E. Malone, and L. S. Symington. 1991. Recombination in yeast, p. 407-522. In E. Jones et al. (ed.), The molecular biology of the yeast Saccharomyces. Cold Spring Harbor Laboratory, Cold Spring Harbor, N.Y.

34. Porter, S. E., and J. J. Champoux. 1989. Mapping in vivo topoisomerase I sites on simian virus 40 DNA: asymmetric distribution of sites on replicating molecules. Mol. Cell. Biol. 9:541-550.

35. Prakash, L., and P. Taillon-Miller. 1981. Effects of the rad52 gene on sister chromatid recombination in Saccharomyces cerevisiae. Curr. Genet. 3:247-250.

36. Prakash, S., L. Prakash, W. Burke, and B. Montelone. 1980. Effect of the RAD52 gene on recombination in Saccharomyces cerevisiae. Genetics 94:31-50.

37. Resnick, M. A. 1987. Investigating the genetic control of biochemical events in meiotic recombination, p. 157-210. In P. Moens (ed.), Meiosis. Academic Press, New York.

38. Reynolds, P., L. Prakash, and S. Prakash. 1987. Nucleotide sequence and functional analysis of the RADl gene of Saccharomyces cerevisiae. Mol. Cell. Biol. 7:1012-1020.

39. Rose, M. D., F. Winston, and P. Hieter. 1990. Methods in yeast genetics, a laboratory manual. Cold Spring Harbor Laboratory Press, Cold Spring Harbor, N.Y.

40. Rothstein, R. J. 1983. One step gene disruption in yeast. Methods Enzymol. 101:202-211.

41. Sambrook, J., E. F. Fritsch, and T. Maniatis. 1989. Molecular 
cloning: a laboratory manual, 2nd ed. Cold Spring Harbor Laboratory Press, Cold Spring Harbor, N.Y.

42. Schiestl, R. H. 1986. Heterothallic mating-type switching in Saccharomyces cerevisiae is RAD52 dependent. Mol. Gen. Genet. 204:496-504.

43. Schiestl, R. H., M. Dominska, and T. D. Petes. 1993. Transformation of Saccharomyces cerevisiae with nonhomologous DNA: illegitimate integration of transforming DNA into yeast chromosomes, and in vivo ligation of transforming DNA to mitochondrial DNA sequences. Mol. Cell. Biol. 13:2697-2705.

44. Schiestl, R. H., and R. D. Gietz. 1989. High efficiency transformation of intact yeast cells by single stranded nucleic acids as carrier. Curr. Genet. 16:339-346.

45. Schiestl, R. H., and T. D. Petes. 1991. Integration of DNA fragments by illegitimate recombination in Saccharomyces cerevisiae. Proc. Natl. Acad. Sci. USA 88:7585-7589.

46. Schiestl, R. H., and S. Prakash. 1988. RAD1, an excision repair gene of Saccharomyces cerevisiae, is also involved in recombination. Mol. Cell. Biol. 8:3619-3626.

47. Schiestl, R. H., and S. Prakash. 1990. RAD10, an excision repair gene of Saccharomyces cerevisiae, is involved in the RAD1 pathway of mitotic recombination. Mol. Cell. Biol. 10:2485-2491.

48. Schiestl, R. H., S. Prakash, and L. Prakash. 1990. The SRS2 suppressor of rad6 mutations of Saccharomyces cerevisiae acts by channeling DNA lesions into the RAD52 DNA repair pathway. Genetics 124:817-831.
49. Schiestl, R. H., and U. Wintersberger. 1982. X-ray enhances mating type switching in heterothallic strains of Saccharomyces cerevisiae. Mol. Gen. Genet. 186:512-517.

50. Shinohara, A., H. Ogawa, and T. Ogawa. 1992. Rad51 protein involved in repair and recombination in $S$. cerevisiae is a RecA-like protein. Cell 69:457-470.

51. Simon, J. R., and P. D. Moore. 1990. Transformation and recombination in rad mutants of Saccharomyces cerevisiae. Mol. Gen. Genet. 223:241-246.

52. Sugawara, N., and J. E. Haber. 1992. Characterization of doublestranded break-induced recombination: homology requirements and single-stranded DNA formation. Mol. Cell. Biol. 12:563-575.

53. Thomas, B. J., and R. Rothstein. 1989. The genetic control of direct-repeat recombination in Saccharomyces: the effect of rad52 and radl on mitotic recombination at $G A L 10$, a transcriptionally regulated gene. Genetics 123:725-738.

54. Weiffenbach, B., and J. E. Haber. 1981. Homothallic mating-type switching generates lethal chromosome breaks in rad52 strains of Saccharomyces cerevisiae. Mol. Cell. Biol. 1:522-534.

55. White, C. I., and J. E. Haber. 1990. Intermediates of recombination during mating type switching in Saccharomyces cerevisiae. EMBO J. 9:663-673.

56. Zamb, T. J., and T. D. Petes. 1981. Unequal sister-strand recombination within yeast ribosomal DNA does not require the RAD52 gene product. Curr. Genet. 3:125-132. 